\title{
MRI and CT into Pathology Tumor and Liver Bile Duct
}

\section{Naser Gjonbalaj' ${ }^{1}$, Astrit Hoxhaj'2, Enkelejda Gjonbalaj ${ }^{3}$, Ardian Biçaku1 ${ }^{1}$, Arben Kutllovci,4, Shkëndije Nuza ${ }^{1}$, Behar Hyseni ${ }^{1}$}

\author{
${ }^{1}$ Radiology Clinic, University Clinical Center of Kosova (UCCK), Pristina, Kosovo of Republic \\ ${ }^{2}$ American Hospital nr-2, Tirana, Albania \\ ${ }^{3}$ Central Pharmacy, University Clinical Center of Kosova (UCCK), Pristina, Kosovo of Republic \\ ${ }^{4}$ Faculty of Medicine, "HASAN PRISHTINA" University of Pristina, Pristina, Kosovo of Republic \\ Email: nasergjonbalaj@gmail.com
}

How to cite this paper: Gjonbalaj, N., Hoxhaj, A., Gjonbalaj, E., Biçaku, A., Kutllovci, A., Nuza, S. and Hyseni, B. (2017) MRI and CT into Pathology Tumor and Liver Bile Duct. Open Journal of Radiology, 7, 259-271.

https://doi.org/10.4236/ojrad.2017.74029

Received: November 6, 2017

Accepted: December 8, 2017

Published: December 11, 2017

Copyright (C) 2017 by authors and Scientific Research Publishing Inc. This work is licensed under the Creative Commons Attribution International License (CC BY 4.0).

http://creativecommons.org/licenses/by/4.0/

\section{Open Access}

\begin{abstract}
Background: Tumor pathologies of the liver and bile ducts are relatively commonly diagnosed and the primary goal is to differentiate these lesions in the fastest possible time which determines the apropriate method of treatment. Aims and Objectives: Aim of this study is tracking and diagnostic imaging correlation of tumor pathologies of liver and bile ducts and determining the early diagnostic approach. Data obtained from this study are important for treatment procedures and succes of treatment. All cases with liver tumor pathologies from period 2012-2016 were examined with CT and MRI followed by other complementary imaging methods. Methods: CT examination was performed according to standard triple-phase protocol: non-enhanced phase, arterial phase and porto-venous phase, and in some cases with late phase after 5 minutes. In MRI examinations, standard protocol was performed: coronal T2 single-shot fast spin-echo (coronal T2 SSFs), Axial T2 respiratory-triggered fast spin-echo (axial T2-FRE)/or breath-hold fast-recovery fast spin-echo T2 body coil sizes XL, (axial T2-FRFSE-XL), Axial in-phase/out-of-phase, Axial/ coronal three-dimensional liver acquisition with volume acceleration (3D coronal pre lava). Results: For comparation, only patients with liver focal lesions were included in the study, and and patient were examined with both diagnostic imaging modalities (CT and MRI). 168 patients with liver tumor pathologies and biliary tree tumor pathologies are included in the study. Gender ratio was 85 males and 83 female (M/F ratio 1.03:1). Average age of patients was 58.41 years, (minimum age 1.5 years old and maximum 88 years of age). The most frequent age on diagnosis was 61 - 70 years (total of 49 patients or 29.16\%). Benign tumors were found with 93 patients $(55.35 \%)$ and malignant tumors in 79 patients (47.02\%). Distribution in liver parenchyma
\end{abstract}


was found in 113 patients (67.26\%), while in 49 patients (29.17\%) pathology was found in the biliary tract. The most affected liver segment was the fifth segment in 35 patients $(20.83 \%)$, while the less affected segment was the second liver segment in 8 patients $(4.73 \%)$. most of patients (62 patients or $36.90 \%)$ had more than two affected segments in time of diagnosis. Diagnostic criteria in this study require further future evaluation. Conclusion: Early diagnosis of are tumors remains a real challenge and has great impact in the survival rate of patients. Finally, our study showed that for our country's institutions that there was no significant difference between both CT and MRI modalities in liver focal lesions assessment.

\section{Keywords}

Tumors, CT, MRI, Non-Ionic Contrast, Gadolinium Contrast

\section{Introduction}

Liver tumor pathologies (LTP)_focal lesions in imaging examinations, are known as changes in normal parenchymal structure of liver. Focal lesions can be cystic, solid or complex/mixed in appearance, depending on content of solid and cystic component. Theoretically, benign lesions are solid, well circumscribed with clear contours, whereas malign lesions have ill circumscribed and unclear margins; however in practice, often it is hard to distinguish benign and malign lesion without biopsy. Liver metastases are more often than primary liver carcinomas (hepatoma). Metastases are multiple, mainly peripherally localized and are of different size [1].

Ultrasound, due to overall easy access and low cost, have its benefits in finding liver and biliary tree tumor pathologies and as being as a first-line imaging modality, however development of other imaging modalities, such as multi-detector computed tomography (MDCT) and magnetic resonance imaging (MRI), gives us much more information on analyzing the vascular/perfuse dynamics of liver tumor lesions.

Development of CT imaging has enabled much more information on liver lesions, especially for analyzing vascular dynamics of tumor lesions [2], and for this reason CT, today have primary role among other non-invasive imaging modalities for liver lesion evaluation. Due to its high sensitivity on detecting even small densitometry alterations, many authors believe that most of liver lesions can be identified or excluded with standard CT or MRI imaging of liver.

Liver tumor pathologies (LTP) are reason for joint researches on challenges in gastroenterology and hepatology. Increased utilization of imaging studies has increased detection and treatment of LTP. Imaging is important not only in malign liver lesions, but also in benign and cystic liver lesions, such as: hemangioma, focal nodular hyperplasia, hepatocellular adenoma, and differential diagnosis of liver cystic lesions, according to American College of Gastroenterology, imaging 
findings as sufficient in diagnosing and managing focal/tumor liver pathologies [3].

Due to overall increased clinical use of imaging modalities such as US, CT and MRI, today earlier not known liver lesions are being detected in asymptomatic patients. According to Smithe-Bindman R. et al. from 1996 to 2010 CT utilization has increased three fold (52/1000 patients in 1996, 149/1000 in 2010, 7.8\% annual increase rate), whereas MRI utilization has increase four times (17/1000 in 1996 to $65 / 1000$, or $10 \%$ annual increase rate); Utilization of US has increased two times (134/1000 to 230/1000, 3.9\% annual increase rate), and PET-CT utilization increased from $0.24 / 1000$ patient to $3.6 / 1000$ patients, $57 \%$ annual increase [4].

According to International Cancer Control Union (ICCU) and International Agency for Research on Cancer (IARC) and American Cancer Society, rate of cancer cases caused from unknown causes are as follow:

- $38 \%$ of 456,000 new cases of esophageal cancer from unknown causes.

- $26 \%$ of $1,825,000$ new cases of lung cancer.

- $79 \%$ of $1,677,000$ new cases of breast cancer.

- $23 \%$ of 782,000 new cases of liver cancer.

- $34 \%$ of 952,000 new cases of gastric cancer.

- $95 \%$ of $1,112,000$ new cases of prostate cancer.

- $0 \%$ of 528,000 new cases of cervical cancer.

- $87 \%$ of $1,361,000$ new cases of colorectal cancer [5].

\section{Clinical Findings in LTP and Biliary Tree Tumor Pathologies (BTTP)}

Common clinical finding in patients with LTP and BTTP are: Fatigue, Chills, Abdominal pain, Hepatomegaly, Weight loss, De-compensatory liver symptoms.

Standard laboratory data obtained together with/or prior to diagnostic imaging criteria are as follow: Alpha feto-protein AFP, SGPT, Bilirubin, HbsAg tests, HCV tests.

The guideline was prepared according to the policies of the American College of Gastroenterology and with the guidance of the Practice Parameters Committee divided into LTP and BTTP as following:

Benign: 1) Hemangioma (Hmg), 2) Hepatocellular Adenoma (HCA), 3) Focal Nodular Hyperplasi (FNH), 4) Nodular Regenerative Hyperplasia $(F R H)$, 5) Biliaary Cyst Adenoma $(B C), 6)$ Biliary Cyst Adenocarcinoma $(B C A), 7)$ Hepatic Cysts (Cysts), 8) Polycytosis of The Liver (PCLD), 9) Hydatid Cysts (Hydat).

Malign: 10) Hepatocellular Carcinoma (HCC), 11) Cholangiocarcinoma (CCA), 12) Hepatic Lymphoma (HL), 13) Hepatic Metastasis (MTS).

\section{Materials and Methods}

During September 2012-June 2016, in UCCK, 168 symptomatic patients with LTP or BTTP who had prior US, have been evaluated with MDCT and MRI.

All subject included hat prior US detected pathology/lesions of liver and bi- 
liary tree, and clinical symptoms of epigastric pain, vomiting etc.

Multiphasic MDCT (MDCT 6 slice and 64 slice machines) pre-contrast, arterial phase and portal-venous phase of liver with slices from 1 to $3 \mathrm{~mm}$ have been performed in almost all patients. Contrast material has been administrated (1 $-1.5 \mathrm{ml} / \mathrm{kg}$ body weight) intravenously in cubital vein (flow rate of $2.5 \mathrm{ml} / \mathrm{s}$ ) with automatic injector. 1.5T MRI with dedicated abdominal coil and standard liver sequences has been applied for upper abdomen evaluation. Liver MRI standard protocol sequences pre and post contrast included: localizer, coronal SSFSE T2, Axial FRE T2 respiratory triggered/or FRFSE-XL T2 breath hold, Axial in-phase/out-of-phase, Axial/coronal 3D lava with administration of intravenous contrast material of $0.5 \mathrm{mmol} / \mathrm{ml}$ injected intravenously in doses of $0.1 \mathrm{ml} / \mathrm{kg}$ body weight.

Where possible, biopsy was performed.

\section{Results and Discussion}

In our retrospective study, from 168 patient diagnosed with LTP and BTTP, 93\% had prior US examination and laboratory essays were performed in all patients (AFP, SGPT, Bilirubine, HbsAg test, HCV test) and further evaluation with MDCT and MRI was performed.

In our results, 85 patients were male (51\%) and 83 patients (49\%) were females, Table 1, male/female ratio $\mathrm{M} / \mathrm{F}=1.03$, which differs from other studies [5].

Mean age of patients diagnosed with LTP and BTTP is 58.4 y.o. (1.5 to 88 y.o.) [6].

Most frequent age-group for both genders diagnosed with LTP dhe BTTP is between 61 - 70 y.o. (29.2\%), comparing to other countries such UK where most frequent age-group is 85 - 89 y.o. [7], which shows younger age in our country, that correlates with developing countries according to WHO, as shown in Table 2.

Mean age for male patients was 59.2 (from 1.5 - 84 y.o.).

Mean age for female patients was 62.7 (13 - 88 y.o.).

In our study, 168 patients with LTP and BTTP were grouped based on location of pathology according to segmental distribution in biliary tree.

It was found that 32 patients had prior cholecystectomy [8]. Smoking was other finding found in 110 patients, which is found as risk factor in other studies [9] [10] [11] [12] [13] (Table 3).

According to gender, our results show that:

Male patient results show:

1) Benign pathology in $57 \%$, malign in $43 \%$ of cases.

Table 1. Gender ratio.

\begin{tabular}{cccc}
\hline Gender ratio $\mathbf{M} / \mathbf{F}$ & M & F & Total \\
\hline $\mathrm{Nr}$ & 85 & 83 & 168 \\
$\%$ & $51 \%$ & $49 \%$ & $100 \%$ \\
\hline
\end{tabular}


Table 2. Pathology according to age.

\begin{tabular}{cccc}
\hline & $\mathrm{nr}$ & $\%$ \\
\hline $1-10$ age & 1 & $0.59 \%$ \\
$11-20$ age & 2 & $1.19 \%$ \\
$21-30$ age & 5 & $2.98 \%$ \\
$31-40$ age & 13 & $7.74 \%$ \\
the spread according to age & $41-50$ age & 26 & $15.48 \%$ \\
& $51-60$ age & 32 & $19.64 \%$ \\
$61-70$ age & 49 & $29.17 \%$ \\
$71-80$ age & 35 & $20.83 \%$ \\
$81-90$ age & 5 & $2.98 \%$ \\
mbi 91 age & 0 & \\
total & 168 & \\
\hline
\end{tabular}

Table 3. Number of patients according to liver segment.

\begin{tabular}{ccc}
\hline in the segment of 2 & 8 patients & $5 \%$ \\
in the segment of 3 & 11 patients & $6 \%$ \\
in the segment of 4 & 10 patients & $6 \%$ \\
in the segment of 5 & 35 patients & $21 \%$ \\
in the segment of 6 & 19 patients & $11 \%$ \\
in the segment of 7 & 10 patients & $6 \%$ \\
in the segment of 8 & 13 patients & $8 \%$ \\
$>$ to a segment & 62 patients & $37 \%$ \\
in the bile duct & 49 patients & $29.17 \%$ \\
Smokers & 110 patients & $65.48 \%$ \\
Non smokers & 58 patients & $35.52 \%$ \\
Cholecyst present & 136 patients & $80.95 \%$ \\
Cholecystectomy & 32 patients & $19.05 \%$ \\
\hline
\end{tabular}

2) Positive diagnosis between imaging modalities CT/MRI $=48 \%$ [14].

3) Positivity between diagnostic imaging modalities MRI/CT $=52 \%$ [14].

4) Distribution according LTP and BTTP and metastases found in 24 patients (28.24\%).

5) The most affected liver segment was segment 5, found in 20 patients $(23.53 \%)$ [15].

6) In more than two liver segments pathology was found in 35 patients $(41.18 \%)$.

7) Prior cholecystectomy was present in 17 patient (20\%) [8].

8) Patients with smoking history was present in $78.82 \%$ of male patients [9] [10] [11] [12] [13].

Female patient results show:

1) Benign pathology found in $49 \%$, malign pathology in $51 \%$ of cases. 
2) Positive diagnosis between imaging modalities CT/MRI $=43 \%$ [8].

3) Positivity between diagnostic imaging modalities MRI/CT $=57 \%$ [8].

4) Distribution according LTP and BTTP and metastases found in 29 patients (34.93\%).

5) The most affected liver segment was segment 5, found in 16 patients (19.28\%) [15].

6) In more than two liver segments pathology was found in 37 patients (44.58\%).

7) Prior cholecystectomy was present in 17 patients (20.48\%) [8].

8) Patients with smoking history was present in $63.85 \%$ of male patients. [9] [10] [11] [12] [13].

Number of LTP and BTTP benign and malign cases, and primary and/or secondary LTP and BTTP, and distribution in liver parenchyma is shown in Table 4.

Distribution of LTP and BTTP in both genders and percentages are shown in Table 5. Our results show that overall rate of liver tumor pathologies and biliary tree tumor pathologies are same as found in literature: hemangioma [5] [16], Figure 1; HCA, Figure 2; FNH, Figure 3; NRH, Figure 4; BC [17], Figure 5; BCA [15] Figure 6; Cysts, Figure 7; PCLD Figure 8; Hydatid Cysts, Figure 9; HCC, Figure 10; CCA, Figure 11; Lymphoma, Figure 12; metastases, Figure 13.

Table 4. Number of cases according to histologic type.

\begin{tabular}{ccc}
\hline Beninje & 8 patients & $54 \%$ \\
Malinj & 11 patients & $46 \%$ \\
Primar in liver & 113 patients & $70 \%$ \\
Secondar in liver & 49 patients & $30 \%$ \\
Liver parenchyma & 113 patients & $70 \%$ \\
The bile duct & 49 patients & $29.17 \%$ \\
\hline
\end{tabular}

Table 5. Number of cases in percentage according to pathology.

\begin{tabular}{ccc}
\hline Hmg & 17 patients & $8 \%$ \\
HCA & 10 patients & $5 \%$ \\
FNH & 9 patients & $4 \%$ \\
NRH & 6 patients & $3 \%$ \\
BC & 9 patients & $4 \%$ \\
BCA & 4 patients & $2 \%$ \\
Cysts & 24 patients & $11 \%$ \\
PCLD & 13 patients & $6 \%$ \\
Hydatid Cysts & 12 patients & $5 \%$ \\
HCC & 21 patients & $10 \%$ \\
CCA & 36 patients & $17 \%$ \\
Limphoma & 1 patients & $0 . \%$ \\
Mts & 53 patients & $25 \%$ \\
\hline
\end{tabular}




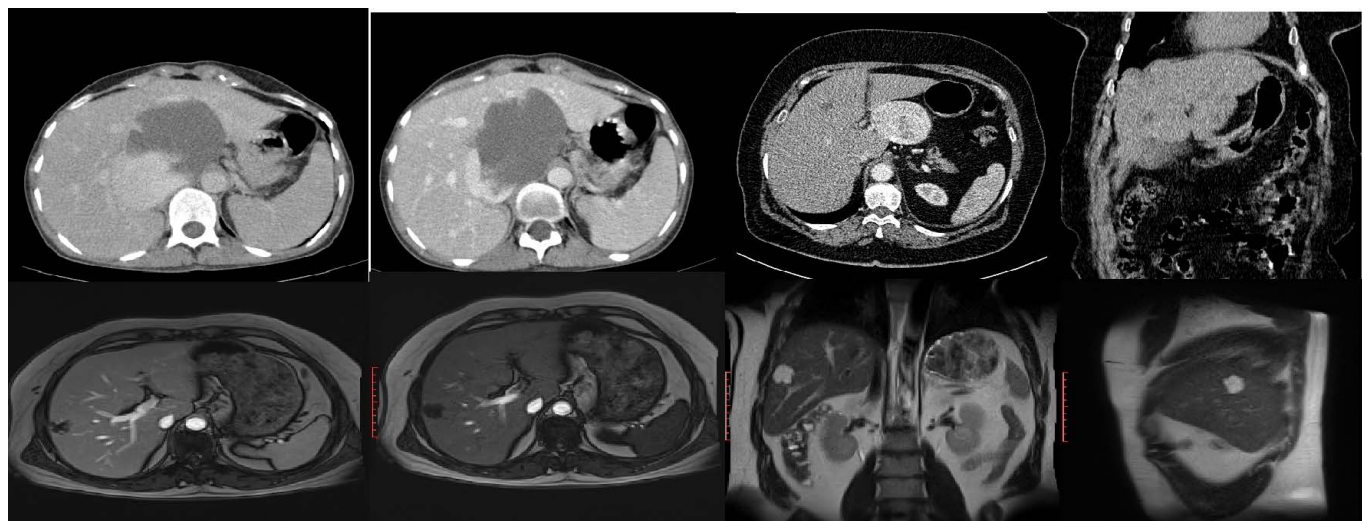

Figure 1. Imaging of hepatic hemangiomas with CT and MR.

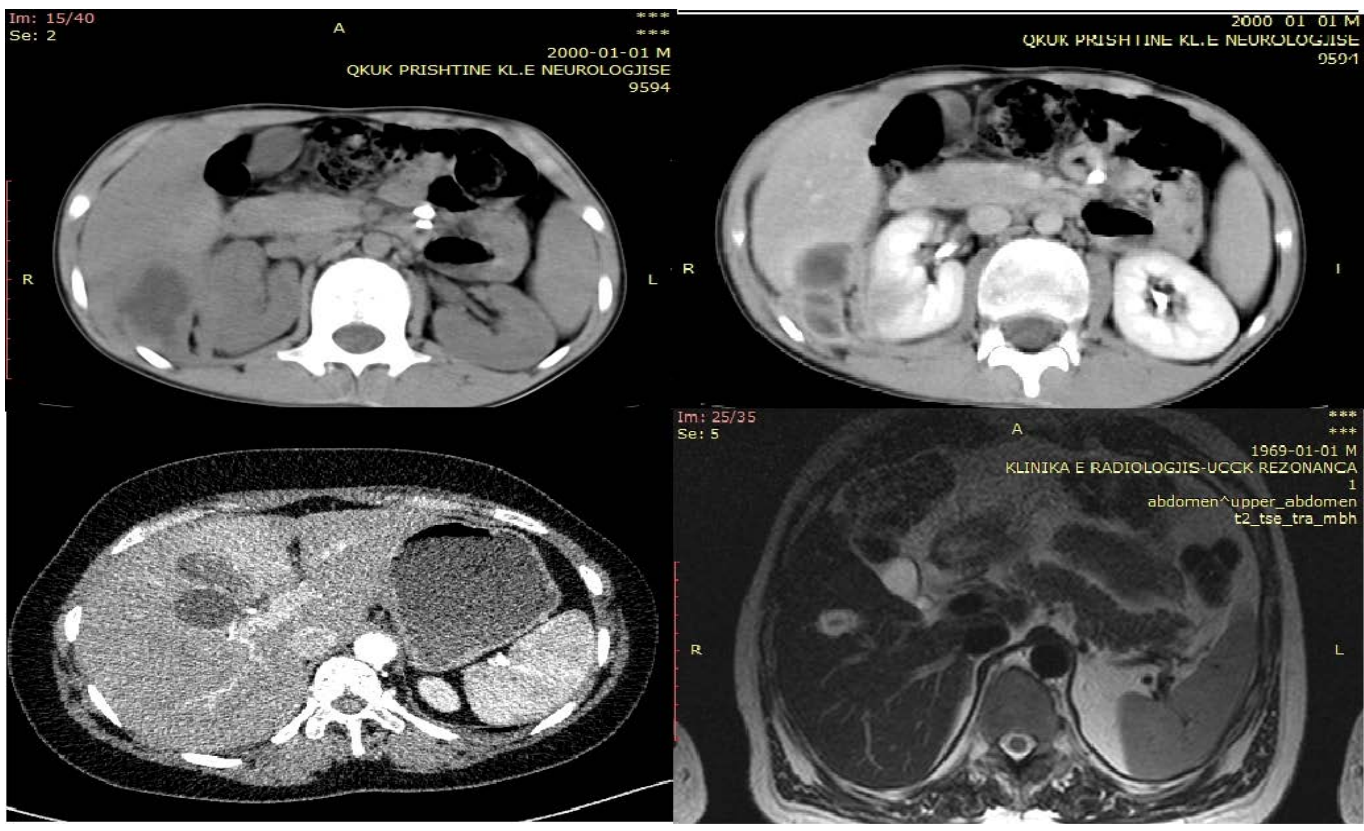

Figure 2. Imaging of hepatic adenomas with CT and MR.

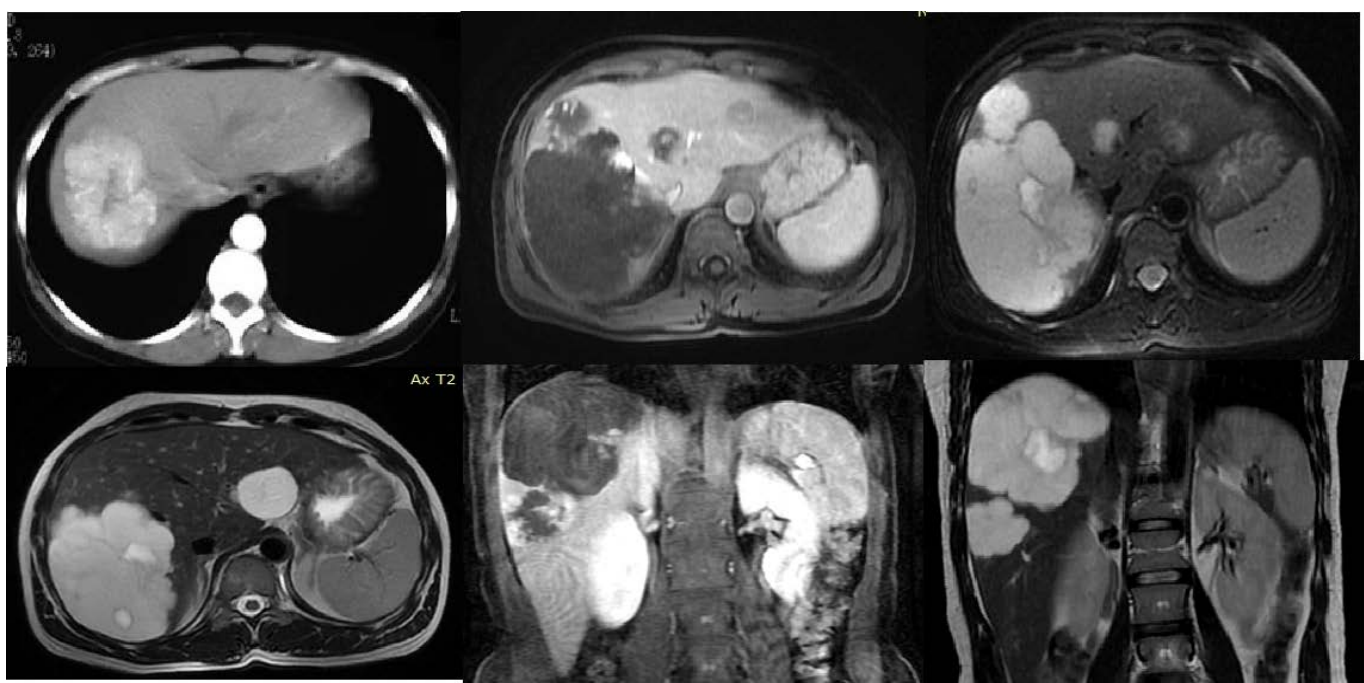

Figure 3. Imaging of FNH with CT and MR. 


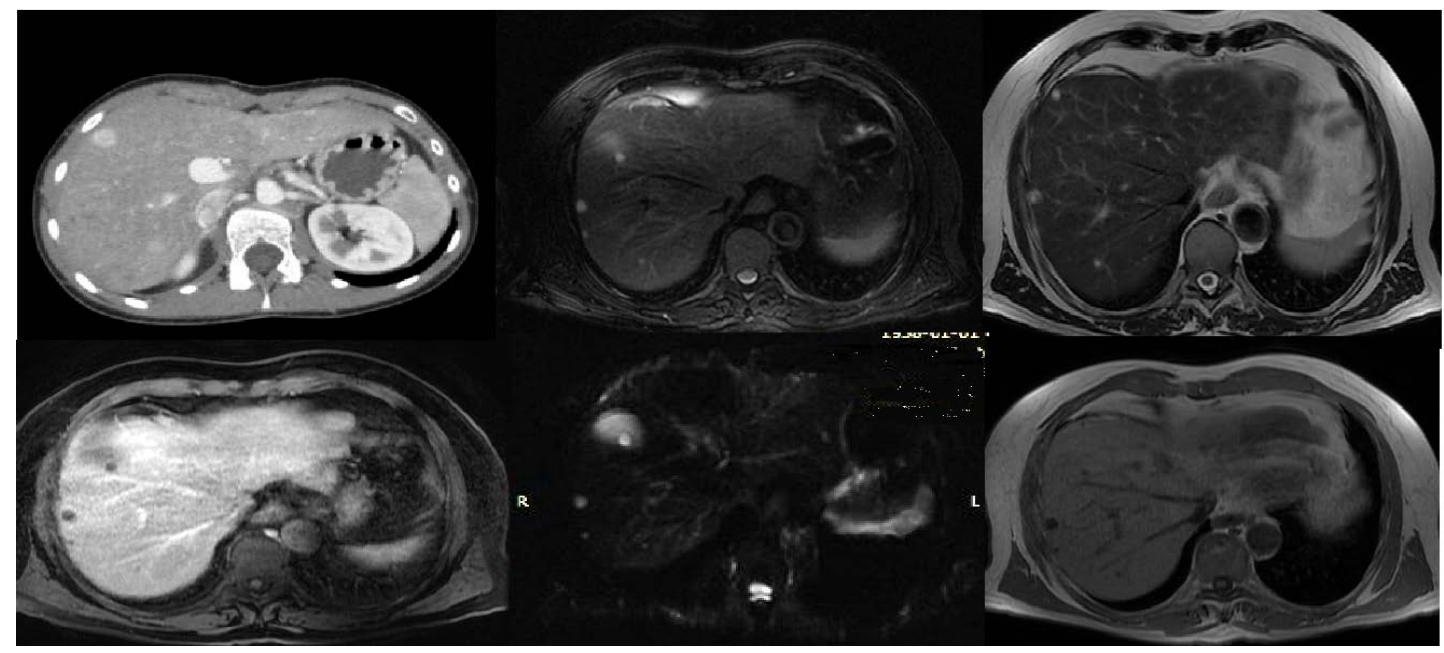

Figure 4. Imaging of FRH with CT and MR.

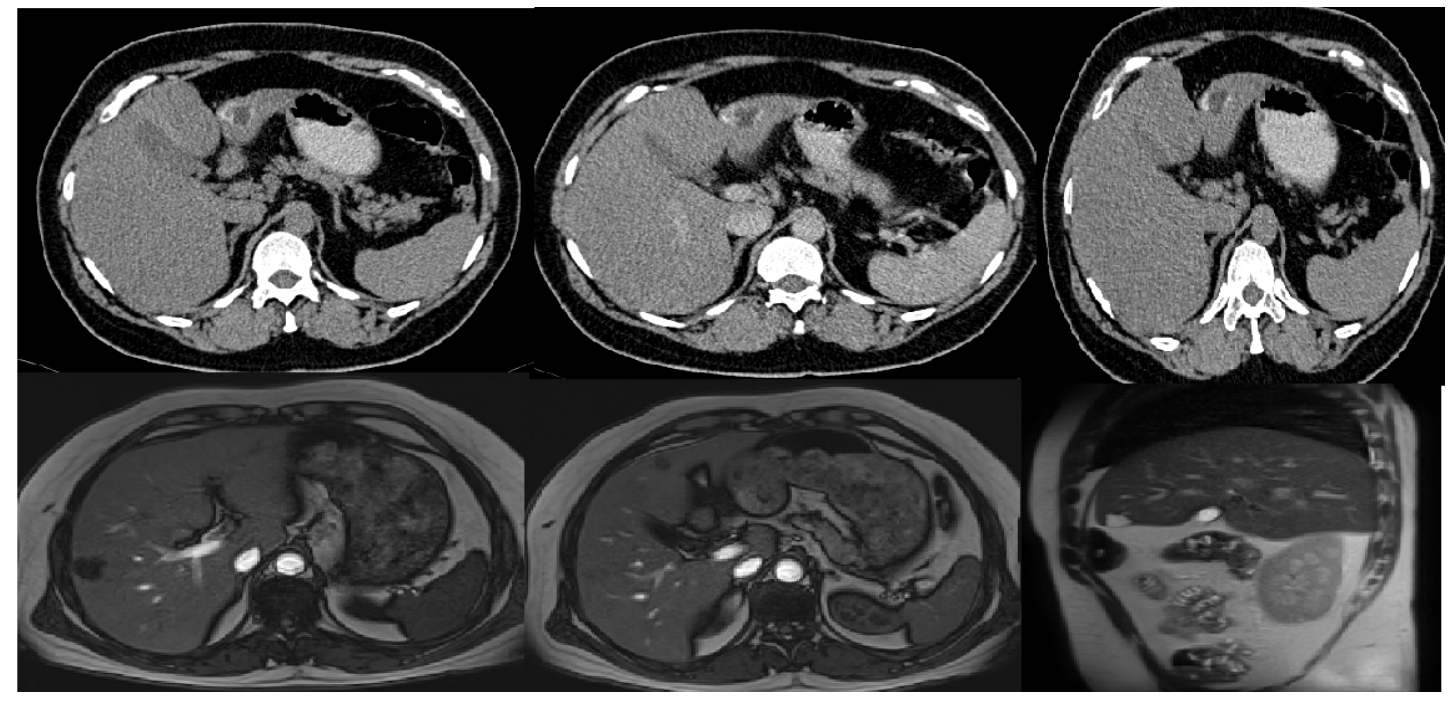

Figure 5. Imaging of bilary cystadenoma with CT and MR.
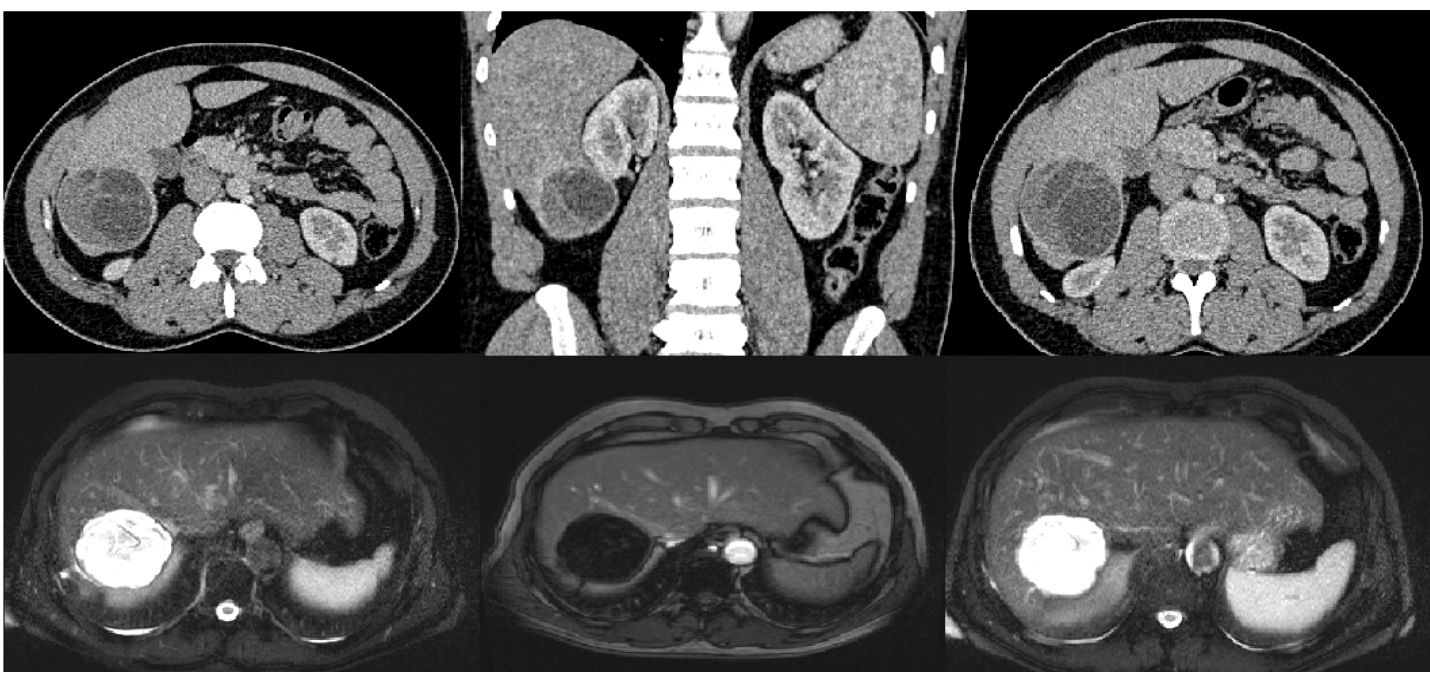

Figure 6. Imaging of Biliary Cystadenocarcinoma with CT and MR. 


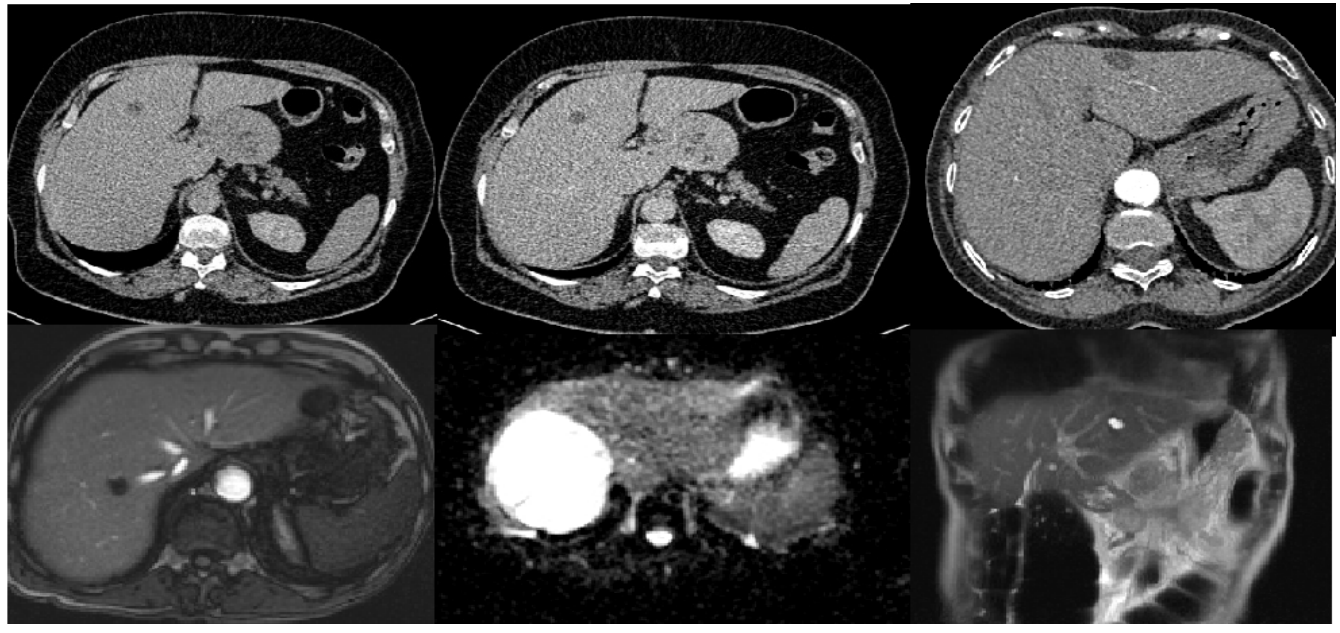

Figure 7. Imaging of Hepatic Cysts with CT and MR.

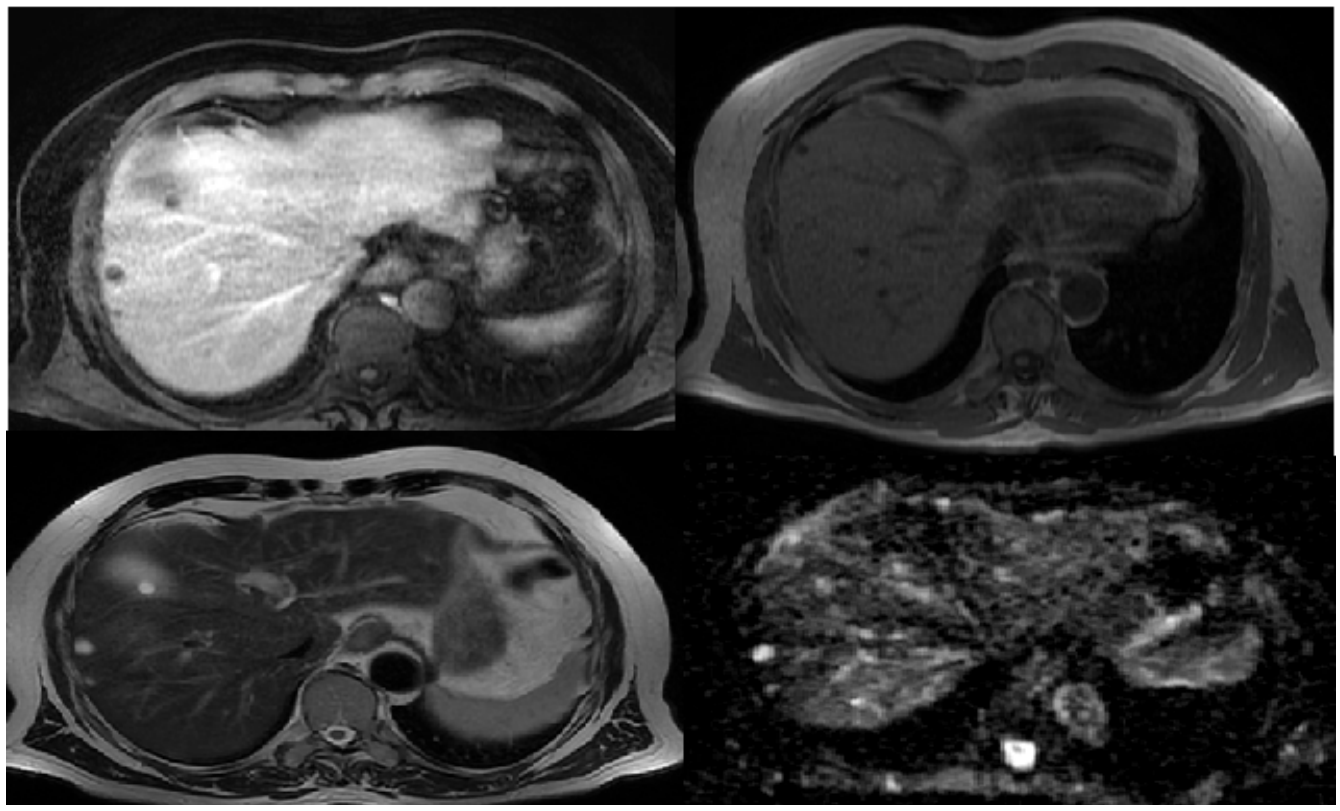

Figure 8. Imaging of Polycytosis of the liver with CT and MR.

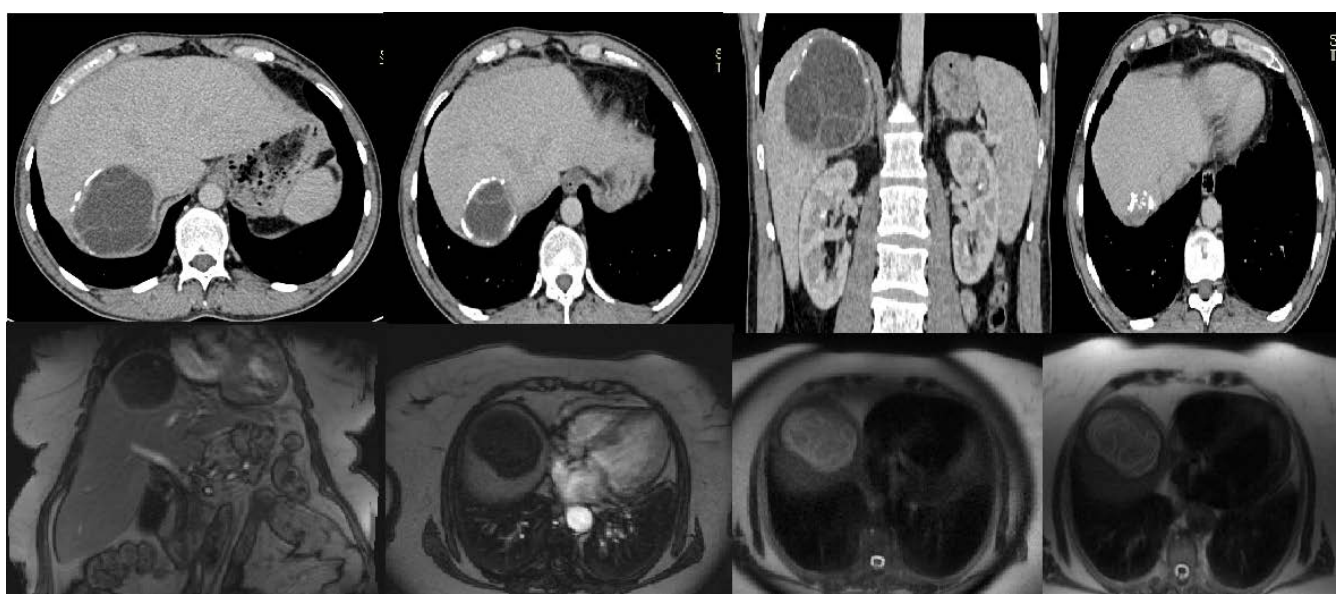

Figure 9. Imaging of Hydatid Cysts with $\mathrm{CT}$ and MR. 


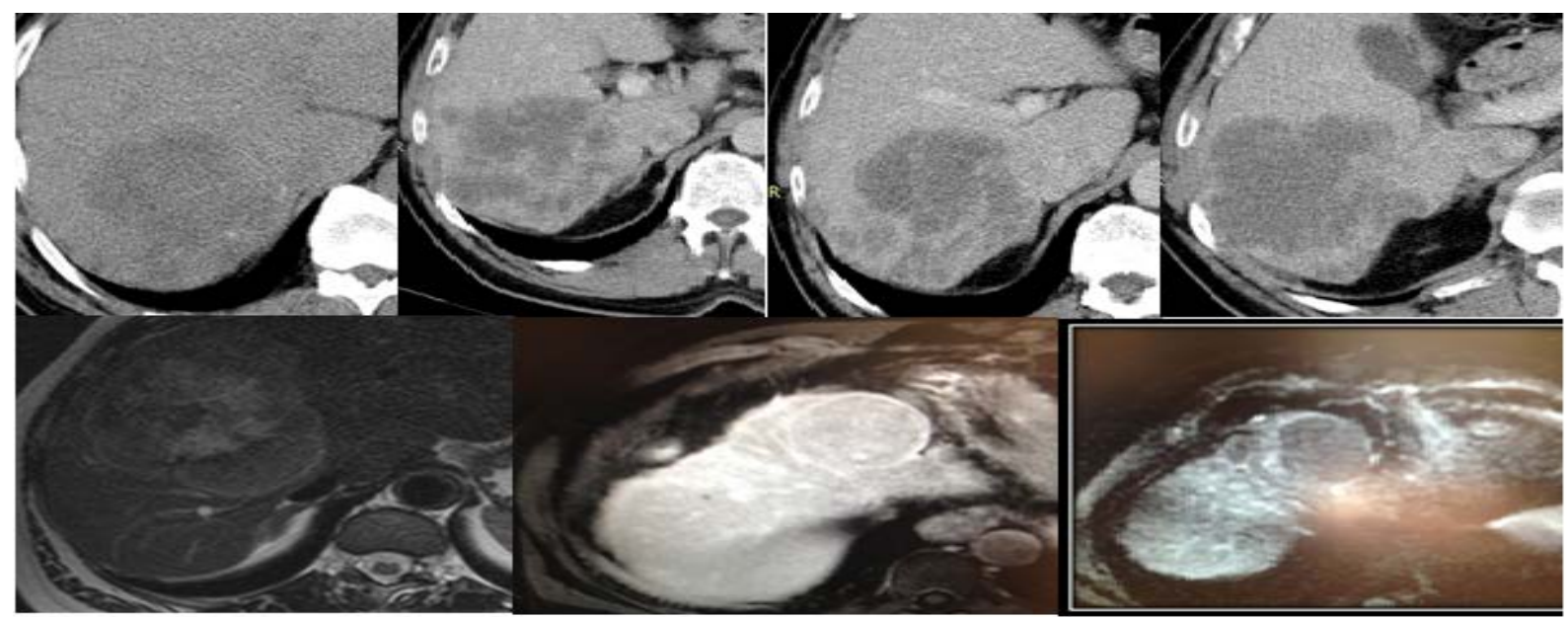

Figure 10. Imaging of Hepatocellular carcinoma with CT and MR.

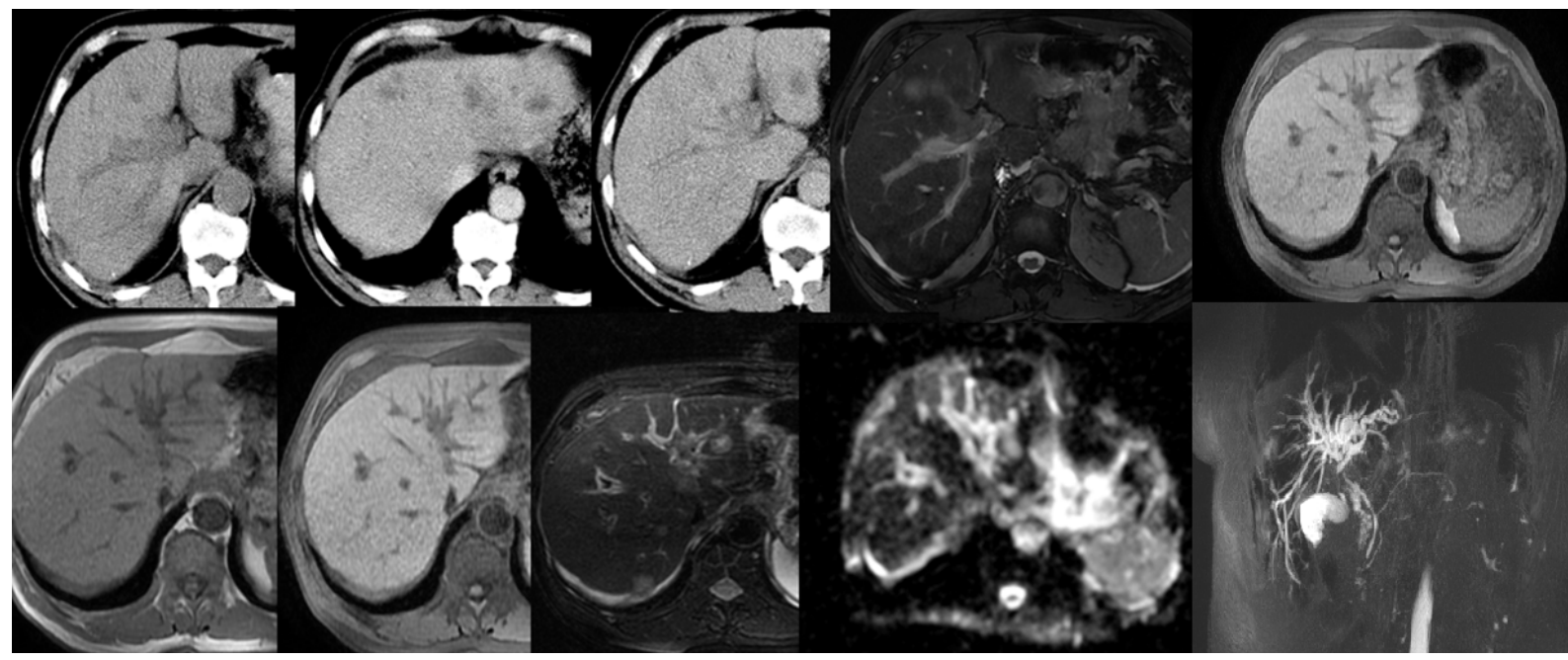

Figure 11. Imaging of Cholangiocarcinoma with CT and MR.

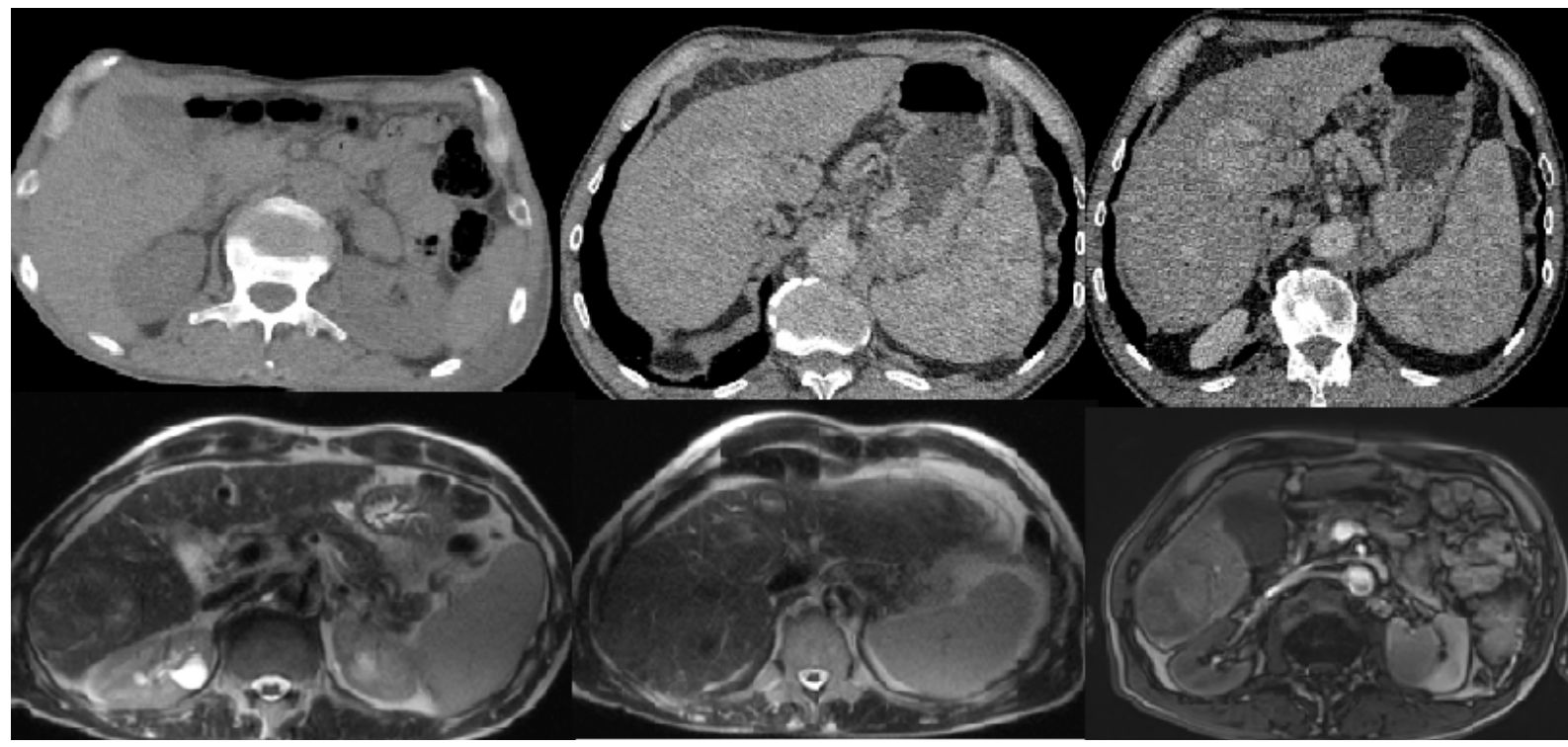

Figure 12. Imaging of Limfoma Hepatike with CT and MR. 


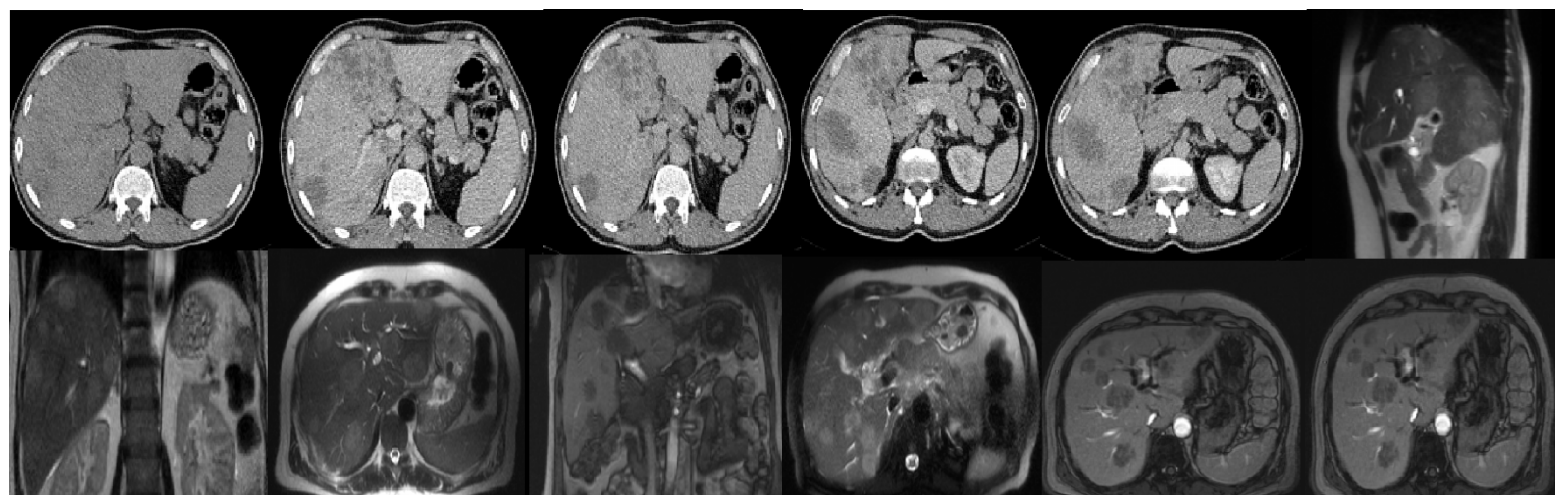

Figure 13. Imaging of liver metastasis with CT and MR.

In recent years the value of dual arterial phase imaging has also been evaluated and is under investigation [18] [19]. In general, CT is less costly than MRI because it involves lower capital equipment costs and a shorter examination time. CT is also widely available and often does not have the same "backlog" of patients as does MRI. Most physicians are relatively comfortable with evaluating CT images themselves, although early all still rely on the interpretation of a diagnostic radiologist for a final diagnosis [20]. The major limitation associated with the use of hepatic CT studies is the need for iodinated contrast material. Both the conventional and the newer nonionic contrast agents are nephrotoxic, and as a result, their use is restricted in patients with renal insufficiency [21] [22]. In addition, CT uses ionizing radiation, the potential harm of which is poorly understood. Most significantly studies have shown that CT is less sensitive and specific than MRI in detecting and characterizing focal hepatic disease [23]. MRI of the liver has several advantages over CT of the liver. MRI provides excellent contrast that can reveal subtle variations in tissues of differing histology. No ionizing radiation or nephrotoxic contrast media is used, and the most commonly administered contrast agents, extracellular gadolinium chelates, have very favorable safety profiles [22]. These advantages of MRI lead to better detection and characterization capabilities than are available with CT. MRI also has some important disadvantage, however.

In most regions, as in our country, it is a more expensive examination than CT. There are fewer MRI scanners than CT scanners, which can result in scheduling difficulties. In general, the MRI examination takes longer and has more contraindications than a CT scan. Finally, MRI has more contraindications than does CT. These include, but are not limited to, the presence of a pacemaker or implanted device (such as a defibrillator or insulin pump), some aneurysm clips and heart valves, and recently placed vascular stents [21].

\section{Conclusions}

In conclusion, our study suggests that:

- There is no significant difference in overall exactness between modalities (CT and MRI) in evaluation of tumor pathologies of liver and biliary tree. 
- Although there is only a small number of patients who underwent MRI evaluation, our study showed higher exactness of MRI than CT.

- CT resulted more exact than MRI in diagnosing hemangiomas, cystic pathologies and metastases.

- CT sensibility in our country is more acceptable imaging modality due to its cost and duration of examination.

- Imaging modalities are complementary to each other, with slight higher positivity of MRI, and are determining for diagnosing and treatment of liver and biliary tree tumor pathologies.

\section{Conflicts of Interest Statement and Funding}

I declare that there is no conflict of interest. This research received no specific grant from any funding agency in the public, commercial, or not-for-profit sectors.

\section{References}

[1] Gjokutajet, A., et al. (2006) Bazat e Ekografise klinike-diagnostike. ASD.

[2] Mc Chesney, E.W. and Hope,J.O. (1957) Studies of the Tissue Distribution and Excretion of Sodium ditriozate in Laboratory Animals. American Journal of Roentgenology, 78, 137-144.

[3] Marrero, et al. (2014) ACG Clinical Guideline: The Diagnosis and Management of Focal Liver Lesions. The American Journal of Gastroenterology, 109, 1328-1347. https://doi.org/10.1038/ajg.2014.213

[4] Smith-Bindman, R., Miglioretti, D.L., Johnson, E., et al. (2012) Use of Diagnostic Imaging Studies and Associated Radiation Exposure for Patients Enrolled in Large integrated Health Care Systems, 1996-2010. JAMA, 307, 2400-2409. https://doi.org/10.1001/jama.2012.5960

[5] (2010) American Cancer Society: AJCC Cancer Staging Manual. 7th Edition, Springer, New York, 191-195.

[6] Fernandez-Pineda, I. and Cabello-Laureano, R. (2014) Differential Diagnosis and Management of Liver Tumors in Infants. World Journal of Hepatology, 6, 486-495. https://doi.org/10.4254/wjh.v6.i7.486

[7] Cancer Research UK, Liver Cancer (C22): 2014. www.cancerresearchuk.org

[8] Coats, M. and Shimi, S.M. (2015) Cholecystectomy and the Risk of Alimentary Tract Cancers: A Systematic Review. World Journal of Gastroenterology, 21, 36793693. https://doi.org/10.3748/wjg.v21.112.3679

[9] Kaltenbach, T.E.-M., Engler, P., Kratzer, W., Oeztuerk, S., Seufferlein, T., Haenle, M.M. and Graeter, T. (2016) Prevalence of Benign Focal Liver Lesions. Abdominal Radiology, 41, 25-32. https://doi.org/10.1007/s00261-015-0605-7

[10] El-Serag, H.B., Engels, E.A., Landgren, O., Chiao, E., Henderson, L., Amaratunge, H.C., et al. (2009) Risk of Hepatobiliary and Pancreatic Cancers after Hepatitis C Virus Infection: A Population-Based Study of U.S. Veterans. Hepatology, 49, 116-123. https://doi.org/10.1002/hep.22606

[11] Shin, H.R., Lee, C.U., Park, H.J., Seol, S.Y., Chung, J.M., Choi, HC., et al. (1996) Hepatitis B and C Virus, Clonorchis Sinensis for the Risk of Liver Cancer: A Case-Control Study in Pusan, Korea. International Journal of Epidemiology, 25, 
933-940. https://doi.org/10.1093/ije/25.5.933

[12] Yamamoto, S., Kubo, S., Hai, S., Uenishi, T., Yamamoto, T., Shuto, T., et al. Hepatitis C Virus Infection as a Likely Etiology of Intrahepatic Cholangiocarcinoma. Cancer Science, 95, 592-595. https://doi.org/10.1111/j.1349-7006.2004.tb02492.x

[13] Tyson, G.L. and El-Serag, H.B. (2011) Risk Factors for Cholangiocarcinoma. Hepatology, 54, 173-184.

[14] Albiin, N. (2012) MRI of Focal Liver Lesions. Current Medical Imaging Reviews, 8, 107-116. https://doi.org/10.2174/157340512800672216

[15] Kanne, J.P., Rohrmann, C.A. and Lichtenstein, J.E. (2006) Eponyms in Radiology of the Digestive Tract: Historical Perspectives and Imaging Appearances. Part 2. Liver, Biliary System, Pancreas, Peritoneum, and Systemic Disease. Radiographics, 26, 465-480. https://doi.org/10.1148/rg.262055130

[16] Souei Mhiri, M., Graiess tlili, K. and Yacoubi, M.T. (2005) Biliary Cystadenocarcinoma: A Case Report Disease. Journal of Radiology, 86, 1035-1037.

[17] Choi, B.I., Lim, J.H., Han, M.C., et al. (1989) Biliary Cystadenoma and Cystadenocarcinoma: CT and Sonographic Findings. Radiology, 171, 57-61. https://doi.org/10.1148/radiology.171.1.2648477

[18] Laghi, A., Iannaccone, R., Rossi, P., et al. (2003) Hepatocellular Carcinoma: Detection with Triple-Phase Multi-Detector Row Helical CT in Patients with Chronic Hepatitis. Radiology, 226, 543-549. https://doi.org/10.1148/radiol.2262012043

[19] Murakami, T., Kim, T., Takamura, M., et al. (2001) Hypervascular Hepatocellular Carcinoma: Detection with Double Arterial Phase Multi-Detector Row Helical CT. Radiology, 218, 763-767. https://doi.org/10.1148/radiology.218.3.r01mr39763

[20] Earls, J.P. (2000) Comparison Studies of CT and MRI in Patients with Hepatic Metastases.

[21] Prince, M.R., Arnoldus, C. and Frisoli, J.K. (1996) Nephrotoxicity of High-Dose Gadolinium Compared with Iodinated Contrast. Journal of Magnetic Resonance Imaging, 6, 162-166. https://doi.org/10.1002/jmri.1880060129

[22] Deray, G., Bellin, M.F., Boulechiar, H., et al. (1991) Nephrotoxicity of Contrast Media in High-Risk Patients with Renal Insufficiency: Comparison of Low- and High-Osmolar Contrast Agents. American Journal of Nephrology, 11, 309-312. https://doi.org/10.1159/000168328

[23] Spring, D.B., Bettmann, M.A. and Barkan, H.E. (1997) Deaths Related to Iodinated Contrast Media Reported Spontaneously to the United States Food and Drug Administration, 1978-1994: Effect of the Availability of Low-Osmolality Contrast Media. Radiology, 204, 333-337. https://doi.org/10.1148/radiology.204.2.9240516 